\title{
Necessary and sufficient condition for the smoothness of intersection local time of subfractional Brownian motions
}

\author{
Guangjun Shen
}

Correspondence: guangjunshen@yahoo.com.cn Department of Mathematics, Anhui Normal University, Wuhu 241000, China

\begin{abstract}
Let $S^{H}$ and $\tilde{S}^{H}$ be two independent $d$-dimensional sub-fractional Brownian motions with indices $H \in(0,1)$. Assume $d \geq 2$, we investigate the intersection local time of subfractional Brownian motions

$$
\ell_{T}=\int_{0}^{T} \int_{0}^{T} \delta\left(S_{t}^{H}-\tilde{S}_{s}^{H}\right) \mathrm{d} s \mathrm{~d} t, \quad T>0,
$$

where $\delta$ denotes the Dirac delta function at zero. By elementary inequalities, we show that $\ell_{T}$ exists in $L^{2}$ if and only if $H d<2$ and it is smooth in the sense of the Meyer-Watanabe if and only if $H<\frac{2}{d+2}$. As a related problem, we give also the regularity of the intersection local time process.
\end{abstract}

2010 Mathematics Subject Classification: 60G15; 60F25; 60G18; 60J55.

Keywords: subfractional Brownian motion, intersection local time, Chaos expansion

\section{Introduction}

The intersection properties of Brownian motion paths have been investigated since the forties (see [1]), and since then, a large number of results on intersection local times of Brownian motion have been accumulated (see Wolpert [2], Geman et al. [3], Imkeller et al. [4], de Faria et al. [5], Albeverio et al. [6] and the references therein). The intersection local time of independent fractional Brownian motions has been studied by Chen and Yan [7], Nualart et al. [8], Rosen [9], Wu and Xiao [10] and the references therein. As for applications in physics, the Edwards' model of long polymer molecules by Brownian motion paths uses the intersection local time to model the 'excluded volume' effect: different parts of the molecule should not be located at the same point in space, while Symanzik [11], Wolpert [12] introduced the intersection local time as a tool in constructive quantum field theory.

Intersection functionals of independent Brownian motions are used in models handling different types of polymers (see, e.g., Stoll [13]). They also occur in models of quantum fields (see, e.g., Albeverio [14]).

As an extension of Brownian motion, recently, Bojdecki et al. [15] introduced and studied a rather special class of self-similar Gaussian processes, which preserves many properties of the fractional Brownian motion. This process arises from occupation time

(c) 2011 Shen; licensee Springer. This is an Open Access article distributed under the terms of the Creative Commons Attribution License (http://creativecommons.org/licenses/by/2.0), which permits unrestricted use, distribution, and reproduction in any medium, provided the original work is properly cited. 
fluctuations of branching particle systems with Poisson initial condition. This process is called the subfractional Brownian motion. The so-called subfractional Brownian motion (sub-fBm in short) with index $H \in(0,1)$ is a mean zero Gaussian process $S^{H}=\left\{S_{t}^{H}, t \geq 0\right\}$ with $S_{0}^{H}=0$ and

$$
C_{H}(s, t):=E\left[S_{t}^{H} S_{s}^{H}\right]=s^{2 H}+t^{2 H}-\frac{1}{2}\left[(s+t)^{2 H}+(t-s)^{2 H}\right]
$$

for all $s, t \geq 0$. For $H=\frac{1}{2}$, $S^{H}$ coincides with the Brownian motion $B$. $S^{H}$ is neither a semimartingale nor a Markov process unless $H=1 / 2$, so many of the powerful techniques from stochastic analysis are not available when dealing with $S^{H}$. The sub-fBm has self-similarity and long-range dependence and satisfies the following estimates:

$$
\left[\left(2-2^{2 H-1}\right) \wedge 1\right](t-s)^{2 H} \leq E\left[\left(S_{t}^{H}-S_{s}^{H}\right)^{2}\right] \leq\left[\left(2-2^{2 H-1}\right) \vee 1\right](t-s)^{2 H} \cdot(1
$$

Thus, Kolmogorov's continuity criterion implies that sub-fBm is Hölder continuous of order $\gamma$ for any $\gamma<H$. But its increments are not stationary. More works for sub$\mathrm{fBm}$ can be found in Bardina and Bascompte [16], Bojdecki et al. [17-19], Shen et al. [20-22], Tudor [23] and Yan et al. [24,25].

In the present paper, we consider the intersection local time of two independent subfBms on $\mathbb{R}^{d}, d \geq 2$, with the same indices $H \in(0,1)$. This means that we have two ddimensional independent centered Gaussian processes $S^{H}=\left\{S_{t}^{H}, t \geq 0\right\}$ and $\tilde{S}^{H}=\left\{\tilde{S}_{t}^{H}, t \geq 0\right\}$ with covariance structure given by

$$
E\left(S_{t}^{H, i} S_{s}^{H, j}\right)=E\left(\tilde{S}_{t}^{H, i} \tilde{S}_{s}^{H, j}\right)=\delta_{i, j} C_{H}(s, t)
$$

where $i, j=1, \ldots, d, s, t \geq 0$. The intersection local time can be formally defined as follows, for every $T>0$,

$$
\ell_{T}=\int_{0}^{T} \int_{0}^{T} \delta\left(S_{t}^{H}-\tilde{S}_{s}^{H}\right) \mathrm{d} s \mathrm{~d} t
$$

where $\delta(\cdot)$ denotes the Dirac delta function. It is a measure of the amount of time that the trajectories of the two processes, $S^{H}$ and $\tilde{S}^{H}$, intersect on the time interval $[0$, $T]$. As we pointed out, this definition is only formal. In order to give a rigorous meaning to $\ell_{T}$, we approximate the Dirac delta function by the heat kernel

$$
p_{\varepsilon}(x)=(2 \pi \varepsilon)^{-\frac{d}{2}} e^{-\frac{|x|^{2}}{2 \varepsilon}}, \quad x \in \mathbb{R}^{d} .
$$

Then, we can consider the following family of random variables indexed by $\varepsilon>0$

$$
\ell_{\varepsilon, T}=\int_{0}^{T} \int_{0}^{T} p_{\varepsilon}\left(S_{t}^{H}-\tilde{S}_{s}^{H}\right) \mathrm{d} s \mathrm{~d} t
$$

that we will call the approximated intersection local time of $S^{H}$ and $\tilde{S}^{H}$. An interesting question is to study the behavior of $\ell_{\varepsilon, T}$ as $\varepsilon$ tends to zero. 
For $H=\frac{1}{2}$, the process $S^{H}$ and $\tilde{S}^{H}$ are Brownian motions. The intersection local time of independent Brownian motions has been studied by several authors (see Wolpert [2], Geman et al. [3] and the references therein). In the general case, that is $H \neq \frac{1}{2}$, only the collision local time has been studied by Yan and Shen [24]. Because of interesting properties of sub-fBm, such as short-/long-range dependence and self-similarity, it can be widely used in a variety of areas such as signal processing and telecommunications( see Doukhan et al. [26]). Therefore, it seems interesting to study the so-called intersection local time for sub-fBms, a rather special class of self-similar Gaussian processes.

The aim of this paper is to prove the existence, smoothness, regularity of the intersection local time of $S^{H}$ and $\tilde{S}^{H}$, for $H \neq \frac{1}{2}$ and $d \geq 2$. It is organized as follows. In Section 2, we recall some facts for the chaos expansion. In Section 3, we study the existence of the intersection local time. In Section 4, we show that the intersection local time is smooth in the sense of the Meyer-Watanabe if and only if $H<\frac{2}{d+2}$. In Section 5 , the regularity of the intersection local time is also considered.

\section{Preliminaries}

In this section, firstly, we recall the chaos expansion, which is an orthogonal decomposition of $L^{2}(\Omega, P)$. We refer to Meyer [27] and Nualart [28] and $\mathrm{Hu}$ [29] and the references therein for more details. Let $X=\left\{X_{b} t \in[0, T]\right\}$ be a $d$-dimensional Gaussian process defined on the probability space $(\Omega, \mathcal{F}, P)$ with mean zero. If $p_{n}\left(x_{1}, \ldots, x_{k}\right)$ is a polynomial of degree $n$ of $k$ variables $x_{1}, \ldots, x_{k}$, then we call $p_{n}\left(X_{t_{1}}^{i_{1}}, \ldots, X_{t_{k}}^{i_{k}}\right)$ a polynomial functional of $X$ with $t_{1}, \ldots, t_{k} \in[0, T]$ and $1 \leq i_{1}, \ldots, i_{k} \leq d$. Let $\mathcal{P}_{n}$ be the completion with respect to the $L^{2}(\Omega, P)$ norm of the set $\left\{p_{m}\left(X_{t_{1}}^{i_{1}}, \ldots, X_{t_{k}}^{i_{k}}\right): 0 \leq m \leq n\right\}$. Clearly, $\mathcal{P}_{n}$ is a subspace of $L^{2}(\Omega, P)$. If $\mathcal{C}_{n}$ denotes the orthogonal complement of $\mathcal{P}_{n-1}$ in $\mathcal{P}_{n}$, then $L^{2}(\Omega, P)$ is actually the direct sum of $\mathcal{C}_{n}$, i.e.,

$$
L^{2}(\Omega, P)=\bigoplus_{n=0}^{\infty} \mathcal{C}_{n}
$$

For $F \in L^{2}(\Omega, P)$, we then see that there exists $F_{n} \in \mathcal{C}_{n}, n=0,1,2, \ldots$, such that

$$
F=\sum_{n=0}^{\infty} F_{n}
$$

This decomposition is called the chaos expansion of $F . F_{n}$ is called the $n$-th chaos of F. Clearly, we have

$$
E\left(|F|^{2}\right)=\sum_{n=0}^{\infty} E\left(\left|F_{n}\right|^{2}\right)
$$

As in the Malliavin calculus, we introduce the space of "smooth" functionals in the sense of Meyer and Watanabe (see Watanabe [30]):

$$
\mathscr{U}:=\left\{F \in L^{2}(\Omega, P): F=\sum_{n=0}^{\infty} F_{n} \quad \text { and } \quad \sum_{n=0}^{\infty} n E\left(\left|F_{n}\right|^{2}\right)<\infty\right\}
$$

and $F \in L^{2}(\Omega, P)$ is said to be smooth if $F \in \mathscr{U}$. 
Now, for $F \in L^{2}(\Omega, P)$, we define an operator $\Upsilon_{u}$ with $u \in[0,1]$ by

$$
\Upsilon_{u} F:=\sum_{n=0}^{\infty} u^{n} F_{n}
$$

Set $\Theta(u):=\Upsilon_{\sqrt{u}} F$. Then, $\Theta(1)=F$. Define $\Phi_{\ominus}(u):=\frac{\mathrm{d}}{\mathrm{d} u}\left(\|\Theta(u)\|^{2}\right)$, where $\|F\|^{2}:=E$ $\left(|F|^{2}\right)$ for $F \in L^{2}(\Omega, P)$. We have

$$
\Phi_{\Theta}(u)=\sum_{n=1}^{\infty} n u^{n-1} E\left(\left|F_{n}\right|^{2}\right) .
$$

Note that $\|\Theta(u)\|^{2}=E\left(|\Theta(u)|^{2}\right)=\sum_{n=1}^{\infty} E\left(u^{n}\left|F_{n}\right|^{2}\right)$.

Proposition 1. Let $F \in L^{2}(\Omega, P)$. Then $F \in \mathscr{U}$, if and only if $\Phi_{\Theta}(1)<\infty$.

Now consider two $d$-dimensional independent sub-fBms $S^{H}$ and $\tilde{S}^{H}$ with indices $H \in$ $(0,1)$. Let $H_{n}(x), x \in \mathbb{R}$ be the Hermite polynomials of degree $n$. That is,

$$
H_{n}(x)=(-1)^{n} \frac{1}{n !} e^{\frac{x^{2}}{2}} \frac{\partial^{n}}{\partial x^{n}} e^{-\frac{x^{2}}{2}} .
$$

Then,

$$
e^{t x-\frac{t^{2}}{2}}=\sum_{n=0}^{\infty} t^{n} H_{n}(x)
$$

for all $t \in \mathbb{C}$ and $x \in \mathbb{R}$, which deduces

$$
\begin{gathered}
\exp \left(i u\left\langle\xi, S_{t}^{H}-\tilde{S}_{s}^{H}\right\rangle+\frac{1}{2} u^{2}|\xi|^{2} \operatorname{Var}\left(S_{t}^{H, 1}-\tilde{S}_{s}^{H, 1}\right)\right) \\
=\sum_{n=0}^{\infty}(i u)^{n} \sigma^{n}(t, s, \xi) H_{n}\left(\frac{\left\langle\xi, S_{t}^{H}-\tilde{S}_{s}^{H}\right\rangle}{\sigma(t, s, \xi)}\right),
\end{gathered}
$$

where $\sigma(t, s, \xi)=\sqrt{\operatorname{Var}\left(S_{t}^{H, 1}-\tilde{S}_{s}^{H, 1}\right)}|\xi|^{2}$ for $\xi \in \mathbb{R}^{d}$. Because of the orthogonality of $\left\{H_{n}(x), x \in \mathbb{R}\right\}_{n \in \mathbb{N}_{+}}$, we will get from (2.2) that

$$
(i u)^{n} \sigma^{n}(t, s, \xi) H_{n}\left(\frac{\left\langle\xi, S_{t}^{H}-\tilde{S}_{s}^{H}\right\rangle}{\sigma(t, s, \xi)}\right)
$$

is the $n$-th chaos of

$$
\exp \left(i u\left\langle\xi, S_{t}^{H}-\tilde{S}_{s}^{H}\right\rangle+\frac{1}{2} u^{2}|\xi|^{2} \operatorname{Var}\left(S_{t}^{H, 1}-\tilde{S}_{s}^{H, 1}\right)\right)
$$

for all $t, s \geq 0$.

\section{Existence of the intersection local time}

The aim of this section is to prove the existence of the intersection local time of $S^{H}$ and $\tilde{S}^{H}$, for an $H \neq \frac{1}{2}$ and $d \geq 2$. We have obtained the following result.

Theorem 2. (i) If $H d<2$, then the $\ell_{\varepsilon, T}$ converges in $L^{2}(\Omega)$. The limit is denoted by $\ell_{T}$ 
(ii) If $H d \geq 2$, then

$$
\lim _{\varepsilon \rightarrow 0} E\left(\ell_{\varepsilon, T}\right)=+\infty,
$$

and

$$
\lim _{\varepsilon \rightarrow 0} \operatorname{Var}\left(\ell_{\varepsilon, T}\right)=+\infty
$$

Note that if $\left\{S_{t}^{\frac{1}{2}}\right\}_{t \geq 0}$ is a planar Brownian motion, then

$$
\ell_{\varepsilon}=\int_{0}^{T} \int_{0}^{T} p_{\varepsilon}\left(S_{t}^{1 / 2}-S_{s}^{1 / 2}\right) \mathrm{d} s \mathrm{~d} t
$$

diverges almost sure, when $\varepsilon$ tends to zero. Varadhan, in [31], proved that the renormalized self-intersection local time defined as $\lim _{\varepsilon \rightarrow 0}\left(\ell_{\varepsilon}-E \ell_{\varepsilon}\right)$ exists in $\mathrm{L}^{2}(\Omega)$. Condition (ii) implies that Varadhan renormalization does not converge in this case.

For $H d \geq 2$, according to Theorem $2, \ell_{\varepsilon, T}$ does not converge in $L^{2}(\Omega)$, and therefore, $\ell_{T}$, the intersection local time of $S^{H}$ and $\tilde{S}^{H}$, does not exist.

Using the following classical equality

$$
p_{\varepsilon}(x)=\frac{1}{(2 \pi \varepsilon)^{\frac{d}{2}}} e^{-\frac{|x|^{2}}{2 e}}=\frac{1}{(2 \pi)^{d}} \int_{\mathbb{R}^{d}} e^{i\langle\xi, x\rangle} e^{-\varepsilon \frac{|\xi|^{2}}{2}} \mathrm{~d} \xi,
$$

we have

$$
\begin{aligned}
\ell_{\varepsilon, T} & =\int_{0}^{T} \int_{0}^{T} p_{\varepsilon}\left(S_{t}^{H}-\tilde{S}_{s}^{H}\right) \mathrm{d} s \mathrm{~d} t \\
& =\frac{1}{(2 \pi)^{d}} \int_{0}^{T} \int_{0}^{T} \int_{\mathbb{R}^{d}} e^{i\left\langle\xi, S_{t}^{H}-\tilde{S}_{s}^{H}\right\rangle} \cdot e^{-\varepsilon \frac{|\varepsilon|^{2}}{2}} \mathrm{~d} \xi \mathrm{d} s \mathrm{~d} t .
\end{aligned}
$$

Since $\left\langle\xi, S_{t}^{H}-\tilde{S}_{s}^{H}\right\rangle \sim N\left(0,|\xi|^{2}\left(2-2^{2 H-1}\right)\left(t^{2 H}+s^{2 H}\right)\right)$, so

$$
E \mid e^{i\left\langle\xi, S_{t}^{H}-\tilde{S}_{s}^{H}\right\rangle}=e^{-\left[\left(2-2^{2 H-1}\right)\left(t^{2 H}+s^{2 H}\right)\right] \frac{|\xi|^{2}}{2}} .
$$

Therefore,

$$
\begin{aligned}
E\left(\ell_{\varepsilon, T}\right) & =\frac{1}{(2 \pi)^{d}} \int_{0}^{T} \int_{0}^{T} \int_{\mathbb{R}^{d}} E\left[e^{i\left\langle\xi, S_{t}^{H}-\tilde{S}_{s}^{H}\right\rangle}\right] \cdot e^{-\varepsilon \frac{|\xi|^{2}}{2}} \mathrm{~d} \xi \mathrm{d} s \mathrm{~d} t \\
& =\frac{1}{(2 \pi)^{d}} \int_{0}^{T} \int_{0}^{T} \int_{\mathbb{R}^{d}} e^{-\left[\varepsilon+\left(2-2^{2 H-1}\right)\left(t^{2 H}+s^{2 H}\right)\right] \frac{|\xi|^{2}}{2}} \mathrm{~d} \xi \mathrm{d} \mathrm{d} t \\
& =\frac{1}{(2 \pi)^{\frac{d}{2}}} \int_{0}^{T} \int_{0}^{T}\left[\varepsilon+\left(2-2^{2 H-1}\right)\left(t^{2 H}+s^{2 H}\right)\right]^{-\frac{d}{2}} \mathrm{~d} s \mathrm{~d} t,
\end{aligned}
$$


where we have used the fact

$$
\int_{\mathbb{R}^{d}} e^{-\left[\varepsilon+\left(2-2^{2 H-1}\right)\left(t^{2 H}+s^{2 H}\right)\right] \frac{|\xi|^{2}}{2}} \mathrm{~d} \xi=\left(\frac{2 \pi}{\varepsilon+\left(2-2^{2 H-1}\right)\left(t^{2 H}+s^{2 H}\right)}\right)^{\frac{d}{2}} .
$$

We also have

$$
\begin{array}{r}
E\left(\ell_{\varepsilon, \mathrm{T}}^{2}\right)=\frac{1}{(2 \pi)^{2 d}} \int_{[0, T]^{4} \mathbb{R}^{2 d}} E\left[e^{i\left\langle\xi, S_{t}^{H}-\tilde{S}_{s}^{H}\right\rangle+i\left\langle\eta, S_{u}^{H}-\tilde{S}_{v}^{H}\right\rangle}\right] \\
\times e^{-\frac{\varepsilon\left(|\xi|^{2}+|\eta|^{2}\right)}{2}} \mathrm{~d} \xi d \eta \mathrm{d} s \mathrm{~d} t \mathrm{~d} u \mathrm{~d} v .
\end{array}
$$

Let we introduce some notations that will be used throughout this paper,

$$
\begin{aligned}
& \lambda_{s, t}=\operatorname{Var}\left(S_{t}^{H, 1}-S_{s}^{H, 2}\right)=\left(2-2^{2 H-1}\right)\left(t^{2 H}+s^{2 H}\right), \\
& \rho_{u, v}=\operatorname{Var}\left(S_{v}^{H, 1}-S_{u}^{H, 2}\right)=\left(2-2^{2 H-1}\right)\left(u^{2 H}+v^{2 H}\right),
\end{aligned}
$$

and

$$
\begin{aligned}
\mu_{s, t, u, v} & =\operatorname{Cov}\left(S_{t}^{H, 1}-S_{s}^{H, 2}, S_{v}^{H, 1}-S_{u}^{H, 2}\right) \\
& =s^{2 H}+t^{2 H}+u^{2 H}+v^{2 H}-\frac{1}{2}\left[(t+v)^{2 H}+|t-v|^{2 H}+(s+u)^{2 H}+|s-u|^{2 H}\right],
\end{aligned}
$$

where $S^{H, 1}$ and $S^{H, 2}$ are independent one dimensional sub-fBms with indices $H$. Using the above notations, we can write for any $\varepsilon>0$

$$
\begin{aligned}
E\left(\ell_{\varepsilon, \mathrm{T}}^{2}\right) & =\frac{1}{(2 \pi)^{2 d}} \int_{[0, T]^{\mathbb{T}} \mathbb{R}^{2 d}} \int_{[0, T]^{4}} \exp \left\{-\frac{1}{2}\left(\left(\lambda_{s, t}+\varepsilon\right)|\xi|^{2}+\left(\rho_{u, v}+\varepsilon\right)|\eta|^{2}+2 \mu_{s, t, u, v}\langle\xi, \eta)\right)\right\} \times \mathrm{d} \xi \mathrm{d} s \mathrm{~d} \mathrm{~d} d u \mathrm{~d} v \\
& =\frac{1}{(2 \pi)^{d}} \int_{[0, t}\left[\left(\lambda_{s, t}+\varepsilon\right)\left(\rho_{u, v}+\varepsilon\right)-\mu_{s, t, u, v}^{2}\right]^{-\frac{d}{2}} \mathrm{~d} s \mathrm{~d} \mathrm{~d} \mathrm{~d} u \mathrm{~d} v .
\end{aligned}
$$

In order to prove the Theorem 2, we need some auxiliary lemmas. Without loss of generality, we may assume $v \leq t, u \leq s$ and $v=x t, u=y s$ with $x, y \in[0,1]$. Then, we can rewrite $\rho_{u, v}$ and $\mu_{s, t, u, v}$ as following.

$$
\begin{gathered}
\rho_{u, v}=\left(2-2^{2 H-1}\right)\left(x^{2 H} t^{2 H}+y^{2 H} s^{2 H}\right), \\
\mu_{s, t, u, v}=t^{2 H}\left\{1+x^{2 H}-\frac{1}{2}\left[(1+x)^{2 H}+(1-x)^{2 H}\right]\right\} \\
+s^{2 H}\left\{1+y^{2 H}-\frac{1}{2}\left[(1+\gamma)^{2 H}+(1-y)^{2 H}\right]\right\} .
\end{gathered}
$$

It follows that

$$
\lambda_{s, t} \rho_{u, v}-\mu_{s, t, u, v}^{2}=t^{4 H} f(x)+s^{4 H} f(y)+t^{2 H} s^{2 H} g(x, y),
$$

where

$$
f(x):=\left(2-2^{2 H-1}\right)^{2} x^{2 H}-\left(1+x^{2 H}-\frac{1}{2}(1+x)^{2 H}-\frac{1}{2}(1-x)^{2 H}\right)^{2},
$$


and

$$
\begin{aligned}
g(x, y)= & \left(2-2^{2 H-1}\right)^{2}\left(x^{2 H}+y^{2 H}\right) \\
& -2\left(1+x^{2 H}-\frac{1}{2}(1+x)^{2 H}-\frac{1}{2}(1-x)^{2 H}\right) \\
& \times\left(1+y^{2 H}-\frac{1}{2}(1+y)^{2 H}-\frac{1}{2}(1-y)^{2 H}\right) .
\end{aligned}
$$

For simplicity throughout this paper, we assume that the notation $F \otimes G$ means that there are positive constants $c_{1}$ and $c_{2}$ so that

$$
c_{1} G(x) \leq F(x) \leq c_{2} G(x)
$$

in the common domain of definition for $F$ and $G$. For $a, b \in \mathbb{R}, a \wedge b:=\min \{a, b\}$ and $a \vee b:=\max \{a, b\}$. By Lemma 4.2 of Yan and Shen [24], we get

Lemma 3. Let $f(x)$ and $g(x, y)$ be defined as above and let $0<H<1$. Then, we have

$$
f(x) \asymp x^{2 H}(1-x)^{2 H} \text {, }
$$

and

$$
g(x, y) \asymp x^{2 H}(1-y)^{2 H}+y^{2 H}(1-x)^{2 H}
$$

for all $x, y \in[0,1]$.

Lemma 4. Let

$$
A_{T}:=\int_{[0, T]^{4}}\left(\lambda_{s, t} \rho_{u, v}-\mu_{s, t, u, v}^{2}\right)^{-\frac{d}{2}} \mathrm{~d} s \mathrm{~d} t \mathrm{~d} u \mathrm{~d} v
$$

Then, $A_{T}$ is finite if and only if $\mathrm{Hd}<2$.

Proof. It is easily to prove the necessary condition. In fact, we can find $\varepsilon>0$ such that $D_{\varepsilon} \subset[0, T]^{4}$, where

$$
D_{\varepsilon} \equiv\left\{(s, t, u, v) \in \mathbb{R}_{+}^{4}: s^{2}+t^{2}+u^{2}+v^{2} \leq \varepsilon^{2}\right\} .
$$

We make a change to spherical coordinates as following

$$
\left\{\begin{array}{l}
s=r \cos \varphi_{1} \\
t=r \sin \varphi_{1} \cos \varphi_{2}, \\
u=r \sin \varphi_{1} \sin \varphi_{2} \cos \varphi_{3} \\
v=r \sin \varphi_{1} \sin \varphi_{2} \sin \varphi_{3} .
\end{array}\right.
$$

where $0 \leq r \leq \varepsilon, 0 \leq \phi_{1}, \phi_{2} \leq \pi, 0 \leq \phi_{3} \leq 2 \pi$,

$$
J=\frac{\partial(s, t, u, v)}{\partial\left(r, \varphi_{1}, \varphi_{2}, \varphi_{3}\right)}=r^{3} \sin ^{2} \varphi_{1} \sin \varphi_{2}
$$

As $\lambda_{s, t} \rho_{u, v}-\mu_{s, t, u, v}^{2}$ is always positive, and $\lambda_{s, t} \rho_{u, v}-\mu_{s, t, u, v}^{2}=r^{4 H} \phi(\theta)$, we have

$$
A_{T} \geq \int_{D_{\varepsilon}}\left(\lambda_{s, t} \rho_{u, v}-\mu_{s, t, u, v}^{2}\right)^{-\frac{d}{2}} \mathrm{~d} s \mathrm{~d} t \mathrm{~d} u \mathrm{~d} v=\int_{0}^{\varepsilon} r^{3-2 H d} \int_{\Theta} \phi(\theta) \mathrm{d} \theta
$$


where the integral in $r$ is convergent if and only if $3-2 H d>-1$ i.e., $H d<2$ and the angular integral is different from zero thanks to the positivity of the integrand. Therefore, $H d \geq 2$ implies that $A_{T}=+\infty$.

Now, we turn to the proof of sufficient condition. Suppose that $H d<2$. By symmetry, we have

$$
A_{T}=4 \int_{\curlyvee}\left(\lambda_{s, t} \rho_{u, v}-\mu_{s, t, u, v}^{2}\right)^{-\frac{d}{2}} \mathrm{~d} s \mathrm{~d} t \mathrm{~d} u \mathrm{~d} v,
$$

where $\Upsilon=f(u, v, s, t): 0<u<s \leq T, 0<v<t \leq T\}$.

By Lemma 3, we get

$$
\begin{aligned}
\lambda_{s, t} \rho_{u, v}-\mu_{s, t, u, v}^{2}= & t^{4 H} f(x)+s^{4 H} f(y)+t^{2 H} s^{2 H} g(x, y) \\
\asymp & t^{4 H} x^{2 H}(1-x)^{2 H}+s^{4 H} y^{2 H}(1-y)^{2 H} \\
& +t^{2 H} s^{2 H}\left(x^{2 H}(1-y)^{2 H}+y^{2 H}(1-x)^{2 H}\right) \\
= & {\left[x^{2 H} t^{2 H}+y^{2 H} s^{2 H}\right]\left[(1-x)^{2 H} t^{2 H}+(1-y)^{2 H} s^{2 H}\right] } \\
= & \left(v^{2 H}+u^{2 H}\right)\left[(t-v)^{2 H}+(s-u)^{2 H}\right] .
\end{aligned}
$$

These deduce for all $H \in(0,1)$ and $T>0$,

$$
\begin{gathered}
\Lambda_{T} \leq C_{H} \int_{0}^{T} \mathrm{~d} t \int_{0}^{t}\left(v^{H}(t-v)^{H}\right)^{-d / 2} \mathrm{~d} v \int_{0}^{T} \mathrm{~d} s \int_{0}^{s}\left(u^{H}(s-u)^{H}\right)^{-d / 2} \mathrm{~d} u \\
=C_{H}\left(\int_{0}^{T} t^{1-H d} \mathrm{~d} t \int_{0}^{1} x^{-\frac{H d}{2}}(1-x)^{-\frac{H d}{2}} \mathrm{~d} x\right)^{2}<\infty .
\end{gathered}
$$

$\square$

Proof of Theorem 2. Suppose $H d<2$, we have

$$
E\left(\ell_{\varepsilon, T} \cdot \ell_{\eta, T}\right)=\frac{1}{(2 \pi)^{d}} \int_{[0, T]^{4}}\left(\left(\lambda_{s, t}+\varepsilon\right)\left(\rho_{u, v}+\eta\right)-\mu_{s, t, u, v}^{2}\right)^{-\frac{d}{2}} \mathrm{~d} s \mathrm{~d} t \mathrm{~d} u \mathrm{~d} v .
$$

Consequently, a necessary and sufficient condition for the convergence in $L^{2}(\Omega)$ of $\ell_{\varepsilon}$, $T$ is that

$$
\int_{[0, T]^{4}}\left(\lambda_{s, t} \rho_{u, v}-\mu_{s, t, u, v}^{2}\right)^{-\frac{d}{2}} \mathrm{~d} s \mathrm{~d} t \mathrm{~d} u \mathrm{~d} v<\infty
$$

This is true due to Lemma 4 .

If $H d \geq 2$, then from (3.2) and using monotone convergence theorem

$$
\lim _{\varepsilon \rightarrow 0} E\left(\ell_{\varepsilon, T}\right)=\frac{1}{\left(2 \pi\left(2-2^{2 H-1}\right)\right)^{d / 2}} \int_{0}^{T} \int_{0}^{T}\left(s^{2 H}+t^{2 H}\right)^{-\frac{d}{2}} \mathrm{~d} s \mathrm{~d} t .
$$

Making a polar change of coordinates

$$
\left\{\begin{array}{l}
x=r \cos \theta \\
y=r \sin \theta
\end{array}\right.
$$


where $0 \geq r \geq T, 0 \leq \theta \leq \frac{\pi}{2}$

$$
\begin{aligned}
& \int_{0}^{T} \int_{0}^{T}\left(s^{2 H}+t^{2 H}\right)^{-\frac{d}{2}} \mathrm{~d} s \mathrm{~d} t \\
& =\int_{0}^{T} \int_{0}^{\frac{\pi}{2}} r^{1-H d}\left(\cos ^{2 H} \theta+\sin ^{2 H} \theta\right)^{-\frac{d}{2}} \mathrm{~d} r \mathrm{~d} \theta
\end{aligned}
$$

and this integral is divergent if $H d \geq 2$. By the expression (3.2) and (3.4), we have

$$
\begin{aligned}
\lim _{\varepsilon \rightarrow 0} \operatorname{Var}\left(\ell_{\varepsilon, T}\right) & =\lim _{\varepsilon \rightarrow 0}\left[E\left(\ell_{\varepsilon, T}^{2}\right)-\left(E \ell_{\varepsilon, T}\right)^{2}\right] \\
& =\frac{1}{(2 \pi)^{d}} \int_{[0, T]^{4}}\left\{\left(\lambda_{s, t} \rho_{u, v}-\mu_{s, t, u, v}^{2}\right)^{-\frac{d}{2}}-\left(\lambda_{s, t} \rho_{u, v}\right)^{-\frac{d}{2}}\right\} \mathrm{d} v \mathrm{~d} u \mathrm{~d} s \mathrm{~d} t .
\end{aligned}
$$

Making a change of variables to spherical coordinates as (3.10), if $H d \geq 2$, we have

$$
\lim _{\varepsilon \rightarrow 0} \operatorname{Var}\left(\ell_{\varepsilon, T}\right)=+\infty
$$

In fact, as the integrand is always positive, we obtain

$$
\begin{aligned}
& \int_{[0, T]^{4}}\left\{\left(\lambda_{s, t} \rho_{u, v}-\mu_{s, t, u, v}^{2}\right)^{-\frac{d}{2}}-\left(\lambda_{s, t} \rho_{u, v}\right)^{-\frac{d}{2}}\right\} \mathrm{d} v \mathrm{~d} u \mathrm{~d} s \mathrm{~d} t \\
\geq & \int_{D_{\varepsilon}}\left\{\left(\lambda_{s, t} \rho_{u, v}-\mu_{s, t, u, v}^{2}\right)^{-\frac{d}{2}}-\left(\lambda_{s, t} \rho_{u, v}\right)^{-\frac{d}{2}}\right\} \mathrm{d} v \mathrm{~d} u \mathrm{~d} s \mathrm{~d} t \\
= & \int_{0}^{\varepsilon} r^{3-2 H d} \mathrm{~d} r \int_{\Theta} \psi(\theta) \mathrm{d} \theta,
\end{aligned}
$$

where the integral in $r$ is convergent if and only if $H d<2$, and the angular integral is different from zero thanks to the positivity of the integrand. Therefore, $H d \geq 2$ implies that

$$
\lim _{\varepsilon \rightarrow 0} \operatorname{Var}\left(\ell_{\varepsilon, T}\right)=+\infty
$$

This completes the proof of Theorem 2 .

\section{Smoothness of the intersection local time}

In this section, we consider the smoothness of the intersection local time. Our main object is to explain and prove the following theorem. The idea is due to An and Yan [32] and Chen and Yan [7].

Theorem 5. Let $\ell_{T}$ be the intersection local time of two independent d-dimensional sub-fBms $S^{H}$ and $\tilde{S}^{H}$ with indices $H L(0,1)$. Then, $\ell_{T} L \mathscr{U}$ if and only if

$$
H<\frac{2}{d+2}
$$


Recall that

$$
\begin{aligned}
& \lambda_{s, t}=\left(2-2^{2 H-1}\right)\left(t^{2 H}+s^{2 H}\right), \\
& \rho_{u, v}=\left(2-2^{2 H-1}\right)\left(u^{2 H}+v^{2 H}\right),
\end{aligned}
$$

and

$$
\mu_{s, t, u, v}=s^{2 H}+t^{2 H}+u^{2 H}+v^{2 H}-\frac{1}{2}\left[(t+v)^{2 H}+|t-v|^{2 H}+(s+u)^{2 H}+|s-u|^{2 H}\right],
$$

for all $s, t, u, v \geq 0$.

In order to prove Theorem 5, we need the following propositions.

Proposition 6. Under the assumptions above, the following statements are equivalent:

(i) $H<\frac{2}{d+2}$;

(ii) $\int_{0}^{T} \int_{0}^{T} \int_{0}^{T} \int_{0}^{T}\left(\lambda_{s, t} \rho_{u, v}-\mu_{s, t, u, v}^{2}\right)^{-\frac{d}{2}-1} \mu_{s, t, u, v}^{2} \mathrm{~d} v \mathrm{~d} u \mathrm{~d} s \mathrm{~d} t<\infty$.

Proof. By (3.12), we have

$$
\begin{aligned}
\lambda_{s, t} \rho_{u, v}-\mu_{s, t, u, v}^{2}= & t^{4 H} f(x)+s^{4 H} f(y)+t^{2 H} s^{2 H} g(x, y) \\
\asymp & t^{4 H} x^{2 H}(1-x)^{2 H}+s^{4 H} y^{2 H}(1-y)^{2 H} \\
& +t^{2 H} s^{2 H}\left(x^{2 H}(1-y)^{2 H}+y^{2 H}(1-x)^{2 H}\right) \\
= & {\left[x^{2 H} t^{2 H}+y^{2 H} s^{2 H}\right]\left[(1-x)^{2 H} t^{2 H}+(1-\gamma)^{2 H} s^{2 H}\right] . }
\end{aligned}
$$

On the other hand, an elementary calculus can show that

$$
x^{2 H} \leq 1+x^{2 H}-\frac{1}{2}(1+x)^{2 H}-\frac{1}{2}(1-x)^{2 H} \leq\left(2-2^{2 H-1}\right) x^{2 H}
$$

for all $x, H \in(0,1)$. By (3.5), we obtain

$$
\left(t^{2 H} x^{2 H}+s^{2 H} y^{2 H}\right)^{2} \leq \mu_{s, t, u, v}^{2} \leq\left(2-2^{2 H-1}\right)^{2}\left(t^{2 H} x^{2 H}+s^{2 H} y^{2 H}\right)^{2} .
$$

It follows that

$$
\begin{aligned}
& \int_{0}^{T} \int_{0}^{T} \int_{0}^{T} \int_{0}^{T}\left(\lambda_{s, t} \rho_{u, v}-\mu_{s, t, u, v}^{2}\right)^{-\frac{d}{2}-1} \mu_{s, t, u, v}^{2} \mathrm{~d} s \mathrm{~d} t \mathrm{~d} u \mathrm{~d} v \\
& \geq C_{H, T} \int_{0}^{T} \int_{0}^{1} \int_{0}^{T} \int_{0}^{1} \frac{\left(t^{2 H} x^{2 H}+s^{2 H} y^{2 H}\right) s t}{\left((1-x)^{2 H} t^{2 H}+(1-\gamma)^{2 H} s^{2 H}\right)^{1+\frac{d}{2}}} \mathrm{~d} \gamma \mathrm{d} s \mathrm{~d} x \mathrm{~d} t \\
& \geq C_{H, T} \int_{0}^{1} \int_{0}^{1} \int_{0}^{1} \int_{0}^{1} \frac{\left(t^{2 H} x^{2 H}+s^{2 H} y^{2 H}\right) s t}{\left((1-x)^{2 H} t^{2 H}+(1-\gamma)^{2 H} s^{2 H}\right)^{1+\frac{d}{2}}} \mathrm{~d} \gamma \mathrm{d} s \mathrm{~d} x \mathrm{~d} t \\
& \geq C_{H, T} \int_{0}^{1} \mathrm{~d} y \int_{0}^{y} \mathrm{~d} x \int_{0}^{x} \mathrm{~d} t \int_{0}^{t H+1} x^{2 H} \frac{t^{2 H}}{t^{2 H(1+d / 2)-1}(1-x)^{2 H(1+d / 2)}} \\
& \geq C_{H, T} \int_{0}^{1} \mathrm{~d} y \int_{0}^{\gamma} \frac{x^{4-H(d-2)}}{(1-x)^{2 H(1+d / 2)} \mathrm{d} x=C_{H, T} \int_{0}^{4-H(d-2)}(1-x)^{1-2 H(1+d / 2)} \mathrm{d} x,}
\end{aligned}
$$


where $C_{H, T}>0$ is a constant depending only on $H$ and $T$ and its value may differ from line to line, which implies that $H<\frac{2}{d+2}$ if the convergence (ii) holds.

On the other hand,

$$
\begin{aligned}
& \int_{0}^{T} \int_{0}^{T} \int_{0}^{T} \int_{0}^{T}\left(\lambda_{s, t} \rho_{u, v}-\mu_{s, t, u, v}^{2}\right)^{-\frac{d}{2}-1} \mu_{s, t, u, v}^{2} \mathrm{~d} u \mathrm{~d} s \mathrm{~d} v \mathrm{~d} t \\
& \leq C_{H} \int_{0}^{T} \int_{0}^{1} \int_{0}^{T} \int_{0}^{1} \frac{\left(t^{2 H} x^{2 H}+s^{2 H} y^{2 H}\right)^{2} s t}{\left[\left(x^{2 H} t^{2 H}+\gamma^{2 H} s^{2 H}\right)\left((1-x)^{2 H} t^{2 H}+(1-\gamma)^{2 H} s^{2 H}\right)\right]^{d / 2+1}} \mathrm{~d} y \mathrm{~d} s \mathrm{~d} x \mathrm{~d} t \\
& \leq C_{H} \int_{0}^{T} \int_{0}^{1} \int_{0}^{T} \int_{0}^{1} \frac{\left(t^{2 H} x^{2 H}+s^{2 H} y^{2 H}\right)^{2} s t}{\left[\left(x^{H} t^{H} y^{H} s^{H}\right)\left((1-x)^{H} t^{H}(1-\gamma)^{H} s^{H}\right)\right]^{d / 2+1}} \mathrm{~d} y \mathrm{~d} s \mathrm{~d} x \mathrm{~d} t \\
& \leq C_{H} \int_{0}^{T} \int_{0}^{1} \int_{0}^{T} \int_{0}^{1} \frac{T^{4 H}}{x^{\frac{d+2}{2} H} y^{\frac{d+2}{2} H}(1-x)^{\frac{d+2}{2} H}(1-\gamma)^{\frac{d+2}{2} H} t^{(d+2) H-1_{s}(d+2) H-1}} \mathrm{~d} y \mathrm{~d} s \mathrm{~d} x \mathrm{~d} t \\
& <\infty
\end{aligned}
$$

if $H<\frac{2}{d+2}$. Where $C_{H}>0$ is a constant depending only on $H$ and its value may differ from line to line. Thus, the proof is completed. $\quad$ व

Hence, Theorem 5 follows from the next proposition.

Proposition 7. Under the assumptions above, the following statements are equivalent: $\ell_{T} \in \mathscr{U}$ if and only if

$$
\int_{0}^{T} \int_{0}^{T} \int_{0}^{T} \int_{0}^{T}\left(\lambda_{s, t} \rho_{u, v}-\mu_{s, t, u, v}^{2}\right)^{-\frac{d}{2}-1} \mu_{s, t, u, v}^{2} \mathrm{~d} u \mathrm{~d} v \mathrm{~d} s \mathrm{~d} t<\infty
$$

In order to prove Proposition 7, we need some preliminaries(see Nualart [28]). Let $X$, $Y$ be two random variables with joint Gaussian distribution such that $E(X)=E(Y)=0$ and $E\left(X^{2}\right)=E\left(Y^{2}\right)=1$. Then, for all $n, m \geq 0$, we have

$$
E\left(H_{n}(X) H_{m}(Y)\right)= \begin{cases}0, & m \neq n \\ \frac{1}{n !}[E(X Y)]^{n}, & m=n\end{cases}
$$

Moreover, elementary calculus can show that the following lemma holds.

Lemma 8 ([7]). Suppose $d \geq 1$. For any $x \in[-1,1)$ we have

$$
\sum_{n=1}^{\infty} \sum_{\substack{k_{1}, \ldots, k_{d}=0 \\ k_{1}+\cdots+k_{d}=n}}^{n} \frac{2 n\left(2 k_{1}-1\right) ! ! \cdots \cdots\left(2 k_{d}-1\right) ! !}{\left(2 k_{1}\right) ! ! \cdots \cdots\left(2 k_{d}\right) ! !} x^{n} \asymp x(1-x)^{-\left(\frac{d}{2}+1\right)} .
$$

Particularly, this is an equality if and only if $d=1$ (see An and Yan [32]).

It follows from $\mu_{s, t, u, v}^{2} \leq \lambda_{s, t} \rho_{u, v}$ that

$$
\begin{aligned}
\frac{\mu_{s, t u, v}^{2}}{\left(\lambda_{s, t} \rho_{u, v}-\mu_{s, t u, v}^{2}\right)^{\frac{d}{2}+1}} & =\frac{\mu_{s, t, u, v}^{2}}{\lambda_{s, t} \rho_{u, v}}\left(1-\frac{\mu_{s, t u, v}^{2}}{\lambda_{s, t} \rho_{u, v}}\right)^{-\left(\frac{d}{2}+1\right)}\left(\frac{1}{\lambda_{s, t} \rho_{u, v}}\right)^{\frac{d}{2}} \\
& \asymp \sum_{\substack { n=1 \\
\begin{subarray}{c}{k_{1}, \ldots, k_{d}=0 \\
k_{1}+\cdots+k_{d}=n{ n = 1 \\
\begin{subarray} { c } { k _ { 1 } , \ldots , k _ { d } = 0 \\
k _ { 1 } + \cdots + k _ { d } = n } }\end{subarray}}^{n} \frac{2 n\left(2 k_{1}-1\right) ! ! \cdots \cdots\left(2 k_{d}-1\right) ! !}{\left(2 k_{1}\right) ! ! \cdots \cdots\left(2 k_{d}\right) ! !} \frac{\mu_{s, t, u, v}^{2 n}}{\left(\lambda_{s, t} \rho_{u, v}\right)^{n+\frac{d}{2}}} .
\end{aligned}
$$


Proof of Proposition 7. For $\varepsilon>0, T \geq 0$, we denote

$$
\Phi_{\Theta_{\varepsilon}}(\kappa):=E\left(\left|\Upsilon_{\sqrt{\kappa}} \ell_{\varepsilon, T}\right|^{2}\right)
$$

and $\Phi_{\Theta}(\kappa):=E\left(\left|\Upsilon_{\sqrt{\kappa}} \ell_{T}\right|^{2}\right)$. Thus, by Proposition 2.1, it suffices to prove (4.3) if and only if $\Phi_{\Theta}(1)<\infty$. Noticing that

$$
\begin{aligned}
\ell_{\varepsilon, T} & =\int_{0}^{T} \int_{0}^{T} p_{\varepsilon}\left(S_{t}^{H}-\tilde{S}_{s}^{H}\right) \mathrm{d} s \mathrm{~d} t \\
& =\frac{1}{(2 \pi)^{d}} \int_{0}^{T} \int_{0}^{T} \int_{\mathbb{R}^{d}} e^{i}\left\langle\xi, S_{t}^{H}-\tilde{S}_{s}^{H}\right\rangle e^{-\varepsilon \frac{|\xi|^{2}}{2}} \mathrm{~d} \xi \mathrm{d} s \mathrm{~d} t \\
& =\frac{1}{(2 \pi)^{d}} \int_{0}^{T} \int_{0}^{T} \int_{\mathbb{R}^{d}} e^{-\frac{1}{2}\left(\lambda_{s, t}+\varepsilon\right)|\xi|^{2}} \sum_{n=0}^{\infty} i^{n} \sigma^{n}(t, s, \xi) H_{n}\left(\frac{\left\langle\xi, S_{t}^{H}-\tilde{S}_{s}^{H}\right\rangle}{\sigma(t, s, \xi)}\right) \mathrm{d} \xi \mathrm{d} s \mathrm{~d} t \\
& \equiv \sum_{n=0}^{\infty} F_{n} .
\end{aligned}
$$

Thus, by (4.4) and Lemma 8 , we have

$$
\begin{aligned}
& \Phi_{\Theta_{\varepsilon}}(1)=\sum_{n=0}^{\infty} n E\left(\left|F_{n}\right|^{2}\right) \\
& =\sum_{n=0}^{\infty} \frac{n}{(2 \pi)^{2 d}} E\left[\int_{[0, T]^{4}} \int_{\mathbb{R}^{2 d}} e^{-\frac{1}{2}\left(\left(\lambda_{s, t}+\varepsilon\right)|\xi|^{2}+\left(\rho_{u, v}+\varepsilon\right)|\eta|^{2}\right)} \sigma^{n}(t, s, \xi) \sigma^{n}(u, v, \eta)\right. \\
& \left.H_{n}\left(\frac{\left\langle\xi, S_{t}^{H}-\tilde{S}_{s}^{H}\right\rangle}{\sigma(t, s, \xi)}\right) H_{n}\left(\frac{\left\langle\eta, S_{u}^{H}-\tilde{S}_{v}^{H}\right\rangle}{\sigma(u, v, \eta)}\right) \mathrm{d} \xi \mathrm{d} \eta \mathrm{d} u \mathrm{~d} v \mathrm{~d} s \mathrm{~d} t\right] \\
& =\sum_{n=1}^{\infty} \frac{1}{(2 \pi)^{2 d}(n-1) !} \int_{[0, T]^{4}} \mu_{s, t, u, v}^{n} \mathrm{~d} u \mathrm{~d} v \mathrm{~d} s \mathrm{~d} t \int_{\mathbb{R}^{2 d}} e^{-\frac{1}{2}\left(\left(\lambda_{s, t}+\varepsilon\right)|\xi|^{2}+\left(\rho_{u, v}+\varepsilon\right)|\eta|^{2}\right)}\langle\xi, \eta\rangle^{n} \mathrm{~d} \xi \mathrm{d} \eta \\
& =\sum_{n=1}^{\infty} \frac{1}{(2 \pi)^{2 d}(2 n-1) !} \int_{[0, T]^{4}} \mu_{s, t, u, v}^{2 n} \mathrm{~d} u \mathrm{~d} v \mathrm{~d} s \mathrm{~d} t \int_{\mathbb{R}^{2 d}} e^{-\frac{1}{2}\left(\left(\lambda_{s, t}+\varepsilon\right)|\xi|^{2}+\left(\rho_{u, v}+\varepsilon\right)|\eta|^{2}\right)}\langle\xi, \eta\rangle^{2 n} \mathrm{~d} \xi \mathrm{d} \eta \\
& =\sum_{n=1}^{\infty} \frac{1}{(2 \pi)^{2 d}(2 n-1) !} \int_{[0, T]^{4}} \mu_{s, t, u, v}^{2 n} \mathrm{~d} u \mathrm{~d} v \mathrm{~d} s \mathrm{~d} t \\
& \times \int_{\mathbb{R}^{2 d}} e^{-\frac{1}{2}\left(\left(\lambda_{s,}+\varepsilon\right)\left(\xi_{1}^{2}+\cdots+\xi_{d}^{2}\right)+\left(\rho_{u, v}+\varepsilon\right)\left(\eta_{1}^{2}+\cdots+\eta_{d}^{2}\right)\right.}\left(\xi_{1} \eta_{1}+\cdots+\xi_{\mathrm{d}} \eta_{d}\right)^{2 n} \mathrm{~d} \xi_{1} \cdots \mathrm{d} \xi_{d} \mathrm{~d} \eta_{1} \ldots \mathrm{d} \eta_{d} \\
& =\sum_{n=1}^{\infty} \frac{1}{(2 \pi)^{2 d}(2 n-1) !} \int_{[0, T]^{4}} \mu_{s, t, u, v}^{2 n} \mathrm{~d} u \mathrm{~d} v \mathrm{~d} s \mathrm{~d} t \times \int_{\mathbb{R}^{2 d}} e^{-\frac{1}{2}\left(\left(\lambda_{s, t}+\varepsilon\right)\left(\xi_{1}^{2}+\cdots+\xi_{d}^{2}\right)+\left(\rho_{u, v}+\varepsilon\right)\left(\eta_{1}^{2}+\cdots+\eta_{d}^{2}\right)\right)} \\
& \sum_{\substack{k_{1}, \ldots, k_{d}=0 \\
k_{1}+\cdots+k_{d}=n}}^{n}\left(\xi_{1} \eta_{1}\right)^{2 k_{1}}\left(\xi_{2} \eta_{2}\right)^{2 k_{2}} \ldots\left(\xi_{\mathrm{d}} \eta_{d}\right)^{2 k_{d}} \mathrm{~d} \xi_{1} \ldots \mathrm{d} \xi_{d} \mathrm{~d} \eta_{1} \ldots \mathrm{d} \eta_{d} \\
& =\frac{1}{(2 \pi)^{d}} \sum_{n=1}^{\infty} \sum_{\substack{k_{1}, \ldots, k_{d}=0 \\
k_{1}+\cdots+k_{d}=n}}^{n} \frac{2 n\left(2 k_{1}-1\right) ! ! \cdots \cdots\left(2 k_{d}-1\right) ! !}{\left(2 k_{1}\right) ! ! \cdots \cdots\left(2 k_{d}\right) ! !} \int_{[0, T]^{4}} \frac{\mu_{s, t, u, v}^{2 n}}{\left(\left(\lambda_{s, t}+\varepsilon\right)\left(\rho_{u, v}+\varepsilon\right)\right)^{n+\frac{d}{2}}} \mathrm{~d} u \mathrm{~d} v \mathrm{~d} s \mathrm{~d} t \\
& \asymp \int_{[0, T]^{4}} \mu_{s, t, u, v}^{2}\left(\left(\lambda_{s, t}+\varepsilon\right)\left(\rho_{u, v}+\varepsilon\right)-\mu_{s, t, u, v}^{2}\right)^{-\frac{d}{2}-1} \mathrm{~d} u \mathrm{~d} v \mathrm{~d} s \mathrm{~d} t
\end{aligned}
$$


where we have used the following fact:

$$
\begin{aligned}
& \int_{\mathbb{R}} \xi^{2 k} e^{-\frac{1}{2}\left(\lambda_{s, t}+\varepsilon\right) \xi^{2}} \mathrm{~d} \xi=2 \int_{0}^{\infty} \xi^{2 k} e^{-\frac{1}{2}\left(\lambda_{s, t}+\varepsilon\right) \xi^{2}} \mathrm{~d} \xi \\
& =2^{k+\frac{1}{2}} \Gamma\left(k+\frac{1}{2}\right)\left(\lambda_{s, t}+\varepsilon\right)^{-\left(k+\frac{1}{2}\right)}=\sqrt{2 \pi}(2 k-1) ! !\left(\lambda_{s, t}+\varepsilon\right)^{-\left(k+\frac{1}{2}\right)} .
\end{aligned}
$$

It follows that

$$
\lim _{\varepsilon \rightarrow 0} \Phi_{\Theta_{\varepsilon}}(1) \asymp \int_{[0, T]^{4}} \mu_{s, t, u, v}^{2}\left(\lambda_{s, t} \rho_{u, v}-\mu_{s, t, u, v}^{2}\right)^{-\frac{d}{2}} \mathrm{~d} u \mathrm{~d} v \mathrm{~d} s \mathrm{~d} t
$$

for all $T \geq 0$. This completes the proof.

\section{Regularity of the intersection local time}

The main object of this section is to prove the next theorem.

Theorem 9. Let $H d<2$. Then, the intersection local time $\ell_{t}$ admits the following estimate:

$$
E\left(\left|\ell_{t}-\ell_{s}\right|^{2}\right) \leq C t^{2-H d}|t-s|^{2-H d}
$$

for a constant $C>0$ depending only on $H$ and $d$.

Proof. Let $C>0$ be a constant depending only on $H$ and $d$ and its value may differ from line to line. For any $0 \leq r, l, u, v \leq T$, denote

$$
\sigma^{2}=\operatorname{Var}\left[\xi\left(S_{r}^{H}-\tilde{S}_{l}^{H}\right)+\eta\left(S_{u}^{H}-\tilde{S}_{v}^{H}\right)\right]
$$

Then, the property of strong local nondeterminism (see Yan et al. [24]):there exists a constant $\kappa_{0}>0$ such that (see Berman [33]) the inequality

$$
\operatorname{Var}\left(\sum_{j=2}^{n} u_{j}\left(S_{t_{j}}^{H}-\tilde{S}_{t_{j-1}}^{H}\right)\right) \geq \kappa_{0} \sum_{j=2}^{n} u_{j}^{2} \operatorname{Var}\left(S_{t_{j}}^{H}-\tilde{S}_{t_{j-1}}^{H}\right) .
$$

holds for $0 \leq t_{1}<t_{2}<\cdots<t_{n} \leq T$ and $u_{j} \in \mathbb{R}, j=2,3, \ldots, n$. and (1.2) yield

$$
\begin{aligned}
\sigma^{2} & =\operatorname{Var}\left(\xi\left(S_{r}^{H}-S_{u}^{H}\right)-\xi\left(\tilde{S}_{l}^{H}-\tilde{S}_{v}^{H}\right)+(\xi+\eta)\left(S_{u}^{H}-\tilde{S}_{v}^{H}\right)\right) \\
& \geq C\left[\xi^{2}\left(|r-u|^{2 H}+|l-v|^{2 H}\right)+(\xi+\eta)^{2}\left(u^{2 H}+v^{2 H}\right)\right] .
\end{aligned}
$$

It follows from (3.1) that for $0 \leq s \leq t \leq T$

$$
\begin{aligned}
E\left|P_{\varepsilon, t}-\ell_{\varepsilon, s}\right|^{2}= & \frac{1}{(2 \pi)^{2 d}} \int_{s}^{t} \int_{s}^{t} \mathrm{~d} r \mathrm{~d} l \int_{s}^{t} \int_{s}^{t} \mathrm{~d} u \mathrm{~d} v \int_{\mathbb{R}^{2 d}} e^{-\frac{1}{2}\left(\sigma^{2}+\varepsilon|\xi|^{2}+\varepsilon|\eta|^{2}\right)} \mathrm{d} \xi \mathrm{d} \eta \\
& +\frac{4}{(2 \pi)^{2 d}} \int_{s}^{t} \mathrm{~d} r \int_{s}^{t} \mathrm{~d} l \int_{s}^{t} \int_{0}^{s} \mathrm{~d} u \mathrm{~d} v \int_{\mathbb{R}^{2 d}} e^{-\frac{1}{2}\left(\sigma^{2}+\varepsilon|\xi|^{2}+\varepsilon|\eta|^{2}\right)} \mathrm{d} \xi \mathrm{d} \eta \\
& +\frac{4}{(2 \pi)^{2 d}} \int_{s}^{t} \mathrm{~d} r \int_{0}^{s} \mathrm{~d} l \int_{s}^{t} \int_{0}^{s} \mathrm{~d} u \mathrm{~d} v \int_{\mathbb{R}^{2 d}} e^{-\frac{1}{2}\left(\sigma^{2}+\varepsilon|\xi|^{2}+\varepsilon|\eta|^{2}\right)} \mathrm{d} \xi \mathrm{d} \eta \\
\equiv & \frac{1}{(2 \pi)^{2 d}}\left[A_{1}(s, t)+4 A_{2}(s, t)+4 A_{3}(s, t)\right] .
\end{aligned}
$$


We have

$$
\begin{aligned}
A_{1}(s, t) & =\int_{s}^{t} \int_{s}^{t} \mathrm{~d} r \mathrm{~d} l \int_{s}^{t} \int_{s}^{t} \mathrm{~d} u \mathrm{~d} v \int_{\mathbb{R}^{2 d}} e^{-\frac{1}{2}\left(\sigma^{2}+\varepsilon|\xi|^{2}+\varepsilon|\eta|^{2}\right)} \mathrm{d} \xi \mathrm{d} \eta \\
& \leq C \int_{s}^{t} \int_{s}^{t} \mathrm{~d} r \mathrm{~d} l \int_{s}^{t} \int_{s}^{t} \mathrm{~d} u \mathrm{~d} v\left[\left(|r-u|^{2 H}+|l-v|^{2 H}\right)\left(u^{2 H}+v^{2 H}\right)\right]^{-\frac{d}{2}} \\
& \leq C \int_{s}^{t} \int_{s}^{t} \int_{s}^{t} \int_{s}^{t}|r-u|^{-\frac{H d}{2}}|l-v|^{-\frac{H d}{2}} u^{-\frac{H d}{2}} v^{-\frac{H d}{2}} \mathrm{~d} r \mathrm{~d} l \mathrm{~d} u \mathrm{~d} v \\
& =C\left(\int_{s}^{t} \int_{s}^{t}|r-u|^{-\frac{H d}{2}} u^{-\frac{H d}{2}} \mathrm{~d} r d u\right)^{2} \leq 4 C\left(\int_{s}^{t} \int_{s}^{r}(r-u)^{-\frac{H d}{2}} u^{-\frac{H d}{2}} d u \mathrm{~d} r\right)^{2},
\end{aligned}
$$

for $0 \leq s \leq t \leq T$. Noting that

$$
\int_{\alpha}^{1}(1-m)^{x-1} m^{x-1} d m \leq \beta_{x}(1-\alpha)^{x}
$$

for all $\alpha \in[0,1]$ and $x>0$, where $\beta_{x}$ is a constant depending only on $x$, we get

$$
\begin{aligned}
\int_{s}^{t} \int_{s}^{r}(r-u)^{-\frac{H d}{2}} u^{-\frac{H d}{2}} d u \mathrm{~d} r & =\int_{s}^{t} r^{1-H d} \mathrm{~d} r \int_{s / r}^{1}(1-m)^{-\frac{H d}{2}} m^{-\frac{H d}{2}} \mathrm{~d} m \\
& \leq C(t-s)^{2-d H}
\end{aligned}
$$

which yields

$$
A_{1}(s, t) \leq C(t-s)^{4-2 d H},
$$

for $0 \leq s \leq t \leq T$. Similarly, for $A_{2}(s, t)$ and $A_{3}(s, t)$ we have also

$$
\begin{aligned}
A_{2}(s, t) & =\int_{s}^{t} \mathrm{~d} r \int_{s}^{t} \mathrm{~d} l \int_{s}^{t} \int_{0}^{s} \mathrm{~d} u \mathrm{~d} v \int_{\mathbb{R}^{2 d}} e^{-\frac{1}{2}\left(\sigma^{2}+\varepsilon|\xi|^{2}+\varepsilon|\eta|^{2}\right)} \mathrm{d} \xi \mathrm{d} \eta \\
& \leq C \int_{s}^{t} \mathrm{~d} r \int_{s}^{t} \mathrm{~d} l \int_{s}^{t} \int_{0}^{s} \mathrm{~d} u \mathrm{~d} v\left[\left(|r-u|^{2 H}+|l-v|^{2 H}\right)\left(u^{2 H}+v^{2 H}\right)\right]^{-\frac{d}{2}} \\
& =C \int_{s}^{t} \int_{s}^{t}|r-u|^{-\frac{H d}{2}} u^{-\frac{H d}{2}} \mathrm{~d} r d u \int_{s}^{t} \mathrm{~d} l \int_{0}^{s}|l-v|^{-\frac{H d}{2}} v^{-\frac{H d}{2}} d v \\
& \leq C t^{2-H d}(t-s)^{2-H d}, \\
A_{3}(s, t) & =\int_{s}^{t} \mathrm{~d} r \int_{0}^{s} \mathrm{~d} l \int_{s}^{t} \int_{0}^{s} \mathrm{~d} u \mathrm{~d} v \int_{\mathbb{R}^{2 d}} e^{-\frac{1}{2}\left(\sigma^{2}+\left.\left.\varepsilon\right|^{2}\right|^{2}+\varepsilon|\eta|^{2}\right)} \mathrm{d} \xi \mathrm{d} \eta \\
& \leq C \int_{s}^{t} \int_{0}^{s} \mathrm{~d} r \mathrm{~d} l \int_{s}^{t} \int_{0}^{t} \mathrm{~d} u \mathrm{~d} v\left[\left(|r-u|^{2 H}+|l-v|^{2 H}\right)\left(u^{2 H}+v^{2 H}\right)\right]^{-\frac{d}{2}} \\
& =C \int_{s}^{t} \int_{s}|r-u|^{-\frac{H d}{2}} u^{-\frac{H d}{2}} \mathrm{~d} r d u \int_{0}^{s} \int_{0}^{s}|l-v|^{-\frac{H d}{2}} v^{-\frac{H d}{2}} \mathrm{~d} l d v \\
& \leq C t^{2-H d}(t-s)^{2-H d},
\end{aligned}
$$


for $0 \leq s \leq t \leq T$. Thus, Theorem 2 and Fatou's lemma yield

$$
E\left(\left|\ell_{t}-\ell_{s}\right|^{2}\right)=E\left(\lim _{\varepsilon \rightarrow 0}\left|\ell_{\varepsilon, t}-\ell_{\varepsilon, s}\right|^{2}\right) \leq \liminf _{\varepsilon \rightarrow 0} E\left(\left|\ell_{\varepsilon, t}-\ell_{\varepsilon, s}\right|^{2}\right) \leq C t^{2-H d}(t-s)^{2-H d} .
$$

This completes the proof. $\quad \square$

\section{Acknowledgements}

The author would like to thank anonymous earnest referee whose remarks and suggestions greatly improved the presentation of the paper. The author is very grateful to Professor Litan Yan for his valuable guidance. This work was supported by National Natural Science Foundation of China (Grant No. 11171062), Key Natural Science Foundation of Anhui Educational Committee (Grant No. KJ2011A139), The Research culture Funds of Anhui Normal University (Grant No. 2010xmpy011) and Natural Science Foundation of Anhui Province.

\section{Competing interests}

The authors declare that they have no competing interests.

Received: 6 September 2011 Accepted: 19 December 2011 Published: 19 December 2011

\section{References}

1. Lévy, P: Le mouvement brownien plan. Am J Math. 62, 487-550 (1940)

2. Wolpert, R: Wiener path intersections and local time. J Funct Anal. 30, 329-340 (1978)

3. Geman, D, Horowitz, J, Rosen, J: A local time analysis of intersections of Brownian paths in the plane. Ann Probab. 12, 86-107 (1984)

4. Imkeller, P, Pérez-Abreu, V, Vives, J: Chaos expansion of double intersection local time of Brownian motion in $\mathbb{\nabla}^{d}$ and renormalization. Stoch Process Appl. 56, 1-34 (1995)

5. de Faria, M, Hida, T, Streit, L, Watanabe, H: Intersection local times as generalized white noise functionals. Acta Appl Math. 46, 351-362 (1997)

6. Albeverio, S, JoÃo Oliveira, M, Streit, L: Intersection local times of independent Brownian motions as generalized White noise functionals. Acta Appl Math. 69, 221-241 (2001)

7. Chen, C, Yan, L: Remarks on the intersection local time of fractional Brownian motions. Stat Probab Lett. 81, 1003-1012 (2011)

8. Nualart, D, Ortiz-Latorre, S: Intersection local time for two independent fractional Brownian motions. J Theor Probab. 20, 759-767 (2007)

9. Rosen, J: The intersection local time of fractional Brownian motion in the plane. J Multivar Anal. 23, 7-46 (1987)

10. Wu, D, Xiao, Y: Regularity of intersection local times of fractional Brownian motions. J Theor Probab. 23, 972-1001 (2010)

11. Symanzik, K: Euclidean quantum field theory. Local Quantum Theory. Academic Press, New York (1969)

12. Wolpert, R: Local time and a particle picture for Euclidean field theory. J Funct Anal. 30, 341-357 (1978)

13. Stoll, A: Invariance principle for Brownian local time and polymer measures. Math Scand. 64, 133-160 (1989)

14. Albeverio, S, Fenstad, JE, Høegh-Krohn, R, Lindstrøm, T: Nonstandard Methods in Stochastic Analysis and Mathematical Physics. Academic Press, New York (1986)

15. Bojdecki, T, Gorostiza, LG, Talarczyk, A: Sub-fractional Brownian motion and its relation to occupation times. Stat Probab Lett. 69, 405-419 (2004)

16. Bardina, X, Bascompte, D: Weak convergence towards two independent Gaussian process from a unique poisson process. Collect Math. 61, 191-204 (2010)

17. Bojdecki, T, Gorostiza, LG, Talarczyk, A: Fractional Brownian density process and its self-intersection local time of order $k$ J. Theor. Probab. 69(5):717-739 (2004)

18. Bojdecki, T, Gorostiza, LG, Talarczyk, A: Limit theorems for occupation time fluctuations of branching systems 1: longrange dependence. Stoch Process Appl. 116, 1-18 (2006)

19. Bojdecki, T, Gorostiza, LG, Talarczyk, A: Some extensions of fractional Brownian motion and sub-fractional Brownian motion related to particle systems. Electron Commun Probab. 12, 161-172 (2007)

20. Shen, G, Yan, L: Remarks on sub-fractional Bessel Precesses. Acta Math Sci. 31B(5):1860-1876 (2011)

21. Shen, G, Yan, L: Remarks on an integral functional driven by sub-fractional Brownian motion. J Korean Stat Soc. 40 , 337-346 (2011)

22. Shen, $\mathrm{G}$, Chen, $\mathrm{C}$ : Stochastic integration with respect to the sub-fractional Brownian motion with $\tilde{S}^{H}$. Stat Probab Lett. 82, 240-251 (2012)

23. Tudor, C: Some properties of the sub-fractional Brownian motion. Stochastics. 79, 431-448 (2007)

24. Yan, L, Shen, G: On the collision local time of sub-fractional Brownian Motions. Stat Probab Lett. 80, 296-308 (2010)

25. Yan, L, Shen, G, He, K: Itô's formula for the subfractional Brownian motion. Commun Stoch Anal. 5, 135-159 (2011)

26. Doukhan, P, Oppenheim, G, Taqqu, MS: Theory and Applications of Long Range Dependence. Birkhäauser, Boston (2003)

27. Meyer, PA: Quantum for Probabilists. Lecture Notes in Mathmatics. Springer, Heidelberg1538 (1993)

28. Nualart, D: Malliavin Calculus and Related Topics. Springer, New York (2006)

29. Hu, YZ: Self-intersection local time of fractional Brownian motion-via chaos expansion. J Math Kyoto Univ. 41, 233-250 (2001)

30. Watanabe, S: Stochastic Differential Equations and Malliavin Calculus. Tata Institute of Fundamental Research. Spring, New York (1984)

31. Varadhan, SRS: Appendix to Euclidean quantum field theory, by K. Symanzik. Local Quantum Theory. Academic Press, New York (1969) 
32. An, L, Yan, L: Smoothness for the collision local time of fractional Brownian motion. Preprint. (2009)

33. Berman, SM: Local nondeterminism and local times of Gaussian processes. Indiana Univ Math J. 23, 69-94 (1973)

doi:10.1186/1029-242X-2011-139

Cite this article as: Shen: Necessary and sufficient condition for the smoothness of intersection local time of subfractional Brownian motions. Journal of Inequalities and Applications 2011 2011:139.

Submit your manuscript to a SpringerOpen ${ }^{\odot}$ journal and benefit from:

- Convenient online submission

- Rigorous peer review

- Immediate publication on acceptance

- Open access: articles freely available online

- High visibility within the field

- Retaining the copyright to your article

Submit your next manuscript at $\gg$ springeropen.com 